\title{
Nierenzellkarzinom: Stabilisierung durch Sequenztherapie
}

— Das progressionsfreie Überleben (PFS) von Patienten mit metastasiertem Nierenzellkarzinom kann durch eine Sequenztherapie signifikant verlängert werden. Am besten belegt ist das für die Behandlung mit Everolimus nach Vortherapie mit einem Tyrosinkinase-Inhibitor (TKI). Bei Versagen einer Erstlinien-Behandlung mit einem TKI und nachdem chirurgische und radiologische Optionen ausgeschöpft sind, stellt sich die Frage, welche systemische Therapie als nächstes folgt: Ein weiterer TKI oder eine Substanz mit anderem Wirkmechanismus? Für die Wahl einer neuen Substanzklasse spricht nach Angaben von Dr. Dirk Arnold, Halle, die Vermutung, dass ein mTOR-Inhibitor wie Everolimus (Afinitor ${ }^{\circledR}$ ) eine TKIResistenz eher durchbricht als ein weiterer TKI. ,Tatsächlich konnte in einer placebokontrollierten Phase-III-Studie die Progredienz der Erkrankung bei TKI-vorbehandelten Patienten mit Everolimus signifikant verzögert werden“, so Arnold. Der Vorteil im progressionsfreien Überleben betrug 4,9 versus 1,9 Monate. Auch das Gesamtüberleben war in der Everolimus-Gruppe länger, wenn das Cross-over von Placebo auf Verum bei der Analyse berücksichtigt wurde.

Die Patienten erreichen mit Everolimus eine Stabilisierung ihrer Erkrankung bei guter Verträglichkeit der oralen Therapie. Höhergradige klinisch relevante Nebenwirkungen sind mit Ausnahme von Hyperglykämien selten., Auch eine erneute Behandlung mit einem TKI ist nachfolgend möglich", so Arnold. Dabei wird entweder auf den primär erfolgreich eingesetzten TKI zurückgegriffen oder ein weiterer TKI in das Sequenzschema eingebaut.

bg

Journalisten-Talk „Herausforderung im Praxisalltag: Welches ist die beste Therapiesequenz beim Nierenzellkarzinom?", Berlin, 29. April 2010

Veranstalter: Novartis, Nürnberg

Schulungsfilme kostenlos zum Download

Fortbildungsfilme sind ein wichtiges Instrument in Hygieneschulungen. Sie zeigen anschaulich und komprimiert, worum es bei den einzelnen Hygienethemen geht und wie sich die geltenden Hygieneanforderungen in die Praxis umsetzen lassen.

In der neu eingerichteten Mediathek auf www.bode-chemie.de stehen ab sofort folgende Schulungsfilme kostenlos zum Download zur Verfügung: ,Hygienische Händedesinfektion “, ,"Chirurgische Händedesinfektion ", ,Hygienische Aufbereitung von flexiblen Endoskopen“ und „Multiresistente Staphylococcus aureus MRSA“. Die Filme spiegeln den aktuellen Stand der Hygieneempfehlungen wider. Der MRSA-Film bietet darüber hinaus je nach Bereich einzeln anwählbare Filme, zum Beispiel zum MRSA-Management in Altenpflege-Einrichtungen.

Nach Informationen von Bode Chemie, Hamburg

\section{Blasenschwäche bei Frauen}

Der Bundesverband für Gesundheitsinformation und Verbraucherschutz - info Gesundheit e.V. (BGV) hat eine Patientenbroschüre zum Thema „Blasenschwäche bei Frauen“ herausgebracht. Der Ratgeber richtet sich ausschließlich an Frauen.

- Die 4-Jahres-Ergebnisse der Combination-of-Avodart-and-Tamsulosin-Studie (CombAT-Studie) zeigen, dass Männer mit moderater bis schwerer BPH (International Prostate Symptom Score [IPSS] $\geq 12$ ) und hohem Progressionsrisiko (Prostatavolumen $\geq 30$ $\mathrm{ml}$ ) unter einer Kombinationstherapie mit dem $5 \alpha$-Reduktasehemmer (5-ARI) Dutasterid und dem $\alpha$-Blocker Tamsulosin (Duod$\operatorname{art}^{\circledR}$ ) signifikant weniger Komplikationen wie akuten Harnverhalt und BPH-bedingte Operationen entwickelten als unter den entsprechenden Monotherapien [Roehrborn CG et al. Eur Urol 2010; 57: 123-31]. Zudem reduzierte diese Kombination rasch, anhaltend und signifikant die Symptomatik sowie das Risiko einer klinischen BPH-Progression gegenüber den Monosubstanzen.

Die Ergebnisse einer aktuellen PosthocAnalyse haben ergeben, dass die Kombinationstherapie mit Dutasterid/Tamsulosin über alle relevanten Risikogruppen hinweg (Prostatavolumen, Symptomatik, PSA-Wert,
Lebensalter und Body-Mass-Index zu Baseline) einer Monotherapie überlegen ist, so Prof. Dr. Stefan Madersbacher, Wien. Die relative Risikoreduktion für das Auftreten von akutem Harnverhalt und/oder der Notwendigkeit einer BPH-bedingten Operation betrug für die Kombinationstherapie gegenüber einer Dutasterid-Monotherapie in den einzelnen Risikogruppen zwischen 5,8 und $44,3 \%$. Weiterhin bestätigen die Daten der Posthoc-Analyse, dass es sich lohnt, schon Patienten ab einem IPSS $<20$ mit der Kombination aus 5-ARI plus $\alpha$-Blocker zu behandeln, da hier die niedrigste Inzidenz von Komplikationen dokumentiert wurde.

aam

Satellitensymposium „The impact of BPH on men's health: New evidence for disease management" im Rahmen des EAU-Kongresses, Barcelona. 17. April 2010

Veranstalter: GlaxoSmithKline, München
Die Broschüre ist auch als Vorbereitung für den Arztbesuch konzipiert. So zeigt sie auf, welche Untersuchungen bei einem Arztbesuches auf die Betroffenen zukommen können. Anhand einer Liste mit typischen Fragen zum Beschwerdebild und zur persönlichen Krankheitsgeschichte können sich die Leserinnen außerdem für das erste Arztgespräch vorbereiten. Neben Grundwissen rund um die Blasenschwäche vermittelt der BGV-Ratgeber auch Tipps zum Alltagsverhalten und zeigt fünf Gymnastikübungen zur Kräftigung des Beckenbodens. Die Broschüre kann kostenlos beim BGV bestellt werden unter www.frauenblasenschwaeche.de.

Nach Informationen des BGV, Bonn 